ITEP-PH-1/2004

\title{
Absorption in Ultra-Peripheral Nucleus-Atom Collisions in Crystal
}

\author{
V.R. Zoller \\ Institute for Theoretical and Experimental Physics \\ 117218 Moscow Russia ${ }^{1}$
}

\begin{abstract}
The Glauber theory description of particle- and nucleus-crystal Coulomb interactions at high-energy is developed. The allowance for the lattice thermal vibrations is shown to produce strong absorption effect which is of prime importance for quantitative understanding of the coherent Coulomb excitation of ultra-relativistic particles and nuclei passing through the crystal.
\end{abstract}

${ }^{1}$ zoller@itep.ru 
In this communication we discuss the origin and estimate the strength of the absorption effect in coherent particle- and nucleus-crystal Coulomb interactions at high-energy.

Generally, the multi-loop corrections generate the imaginary part of the scattering amplitude even if the tree-level amplitude is purely real. For example, the purely real Born amplitude of the high-energy Coulomb scattering in crystal acquires the imaginary part due to the multiple scattering (MS) effects. However, in the widely used static/frozen lattice approximation (SL approximation) the account of rescatterings alters only the overall real phase of the full amplitude thus producing no absorption effect. The latter is related to the creation and annihilation of excited intermediate states of crystal and as such manifests itself only beyond the SL approximation. With the allowance for the lattice thermal vibrations the Coulomb phase shift function gets non-vanishing imaginary part which is interpreted as an absorption effect. The account of the lattice thermal vibrations provides a natural ultra-violet regulator of the theory and, as we shall see, enables quantitative understanding of the phenomenon of the coherent Coulomb excitation of relativistic particles and nuclei passing through the crystal. The latter is the goal we pursue in this communication.

We start with the well known example of the coherent Coulomb elastic scattering of charged particle (charge $Z_{1}$ ) by a linear chain of $N$ identical atoms in a crystal target. The interatomic distances in crystal, $a$, are large, compared to the Thomas-Fermi screening

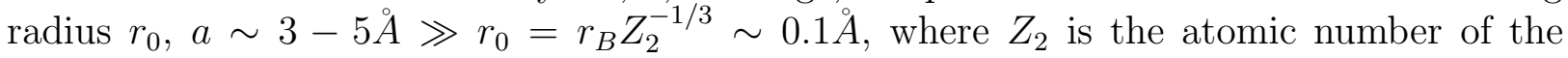
target atom and $r_{B}$ is the Bohr radius [2]. The relevant impact parameters, $b$, satisfy the condition $b \ll a$ and the amplitudes of scattering by different atomic chains parallel to a given crystallographic axis are incoherent.

The amplitude of small-angle elastic scattering in the eikonal approximation [1] can be represented in the following form

$$
F(q)=i p \int b d b J_{0}(q b)\left\{1-\langle\exp [i \chi(\mu b)]\rangle^{N}\right\} .
$$

where $\mathbf{q}$ is the $2 D$-vector of the momentum transfer and the incident particle momentum $p$ is assumed to be large enough to satisfy the condition of applicability of the straight paths approximation, $p / q^{2} \gg a N$. The latter condition insures the coherence of interactions with different atoms. In eq.(1) the screened Coulomb phase shift function is

$$
\chi(\mu b)=-\beta K_{0}(\mu b),
$$

with $\beta=2 \alpha Z_{1} Z_{2}$ and $\mu=r_{0}^{-1}$. Hereafter, $J_{0,1}(x)$ and $K_{0,1}(x)$ are the Bessel functions. The brackets \langle\rangle signify that an average is to be taken over all configurations of atoms in the ground state of crystal. We neglect all position correlations of the atoms and describe the ground state of crystal by the wave function $|\Psi\rangle \propto \prod_{j=1}^{N}\left|\psi_{j}\right\rangle$.

After the azimuthal integration the term $\langle\exp (i \chi)\rangle$ takes the form

$$
\begin{array}{r}
\langle\exp (i \chi)\rangle=\int d^{2} \mathbf{s} \rho(s) \exp [i \chi(\mu|\mathbf{b}-\mathbf{s}|)] \\
=\exp \left(-\Omega^{2} b^{2}\right) \int d x \exp (-x) \\
\times I_{0}(2 b \Omega \sqrt{x}) \exp \left[-i \beta K_{0}(\mu \sqrt{x} / \Omega)\right] .
\end{array}
$$

The $2 D$-vector $\mathbf{s}$, describes the position of the target atom in the impact parameter plane. The one-particle probability distribution $\rho(s)$ is as follows

$$
\rho(s)=\int d z|\psi(\mathbf{s}, z)|^{2}=\left(\Omega^{2} / \pi\right) \exp \left(-\Omega^{2} \mathbf{s}^{2}\right) .
$$


For the most commonly studied elements at room temperature the ratio $\mu / \Omega$ varies in a wide range, from $\mu / \Omega \sim 0.1$ to $\mu / \Omega \sim 1[2]$. Consider first the region of small impact parameters. For $b \ll 1 / 2 \Omega$ only small $s$, such that $\mu s \lesssim 1$, contribute. One can put then in eq. (3) $K_{0}(\mu s) \simeq \log (1 / \mu s)$ and integrate over $s$. The result is

$$
\langle\exp (i \chi)\rangle=\left(\frac{\mu}{\Omega}\right)^{i \beta} \Gamma\left(1+\frac{i \beta}{2}\right) \Phi\left(-\frac{i \beta}{2} ; 1 ;-\Omega^{2} b^{2}\right) .
$$

In eq. (5) $\Phi(a, b ; z)$ is the confluent hyper-geometric function. From (5) it follows, in particular, that

$$
|\langle\exp (i \chi)\rangle|_{b=0}=\left[\frac{\pi \beta}{2 \sinh (\pi \beta / 2)}\right]^{1 / 2}
$$

and in the weak coupling regime, $\beta \ll 1$,

$$
|\langle\exp (i \chi)\rangle|_{b=0} \simeq 1-\frac{\pi^{2} \beta^{2}}{48}
$$

while for $\beta \gtrsim 1$

$$
|\langle\exp (i \chi)\rangle|_{b=0} \simeq \sqrt{\pi \beta} \exp \left(-\frac{\pi \beta}{4}\right)
$$

Therefore, at small impact parameters, $b \lesssim \Omega^{-1}$, the intensity of outgoing nuclear waves as a function of $N$ exhibits the exponential attenuation.

The absorption effect becomes weaker toward the region of large impact parameters $b \gtrsim 1 / 2 \Omega$

$$
\begin{aligned}
& |\langle\exp (i \chi)\rangle|^{N} \simeq|\langle\exp (i \chi)\rangle|_{b=0}^{N} \\
& \quad \times\left[1+\frac{N \beta^{2}}{16}(\Omega b)^{4}+\ldots\right] .
\end{aligned}
$$

For still larger $b, b \gg 1 / 2 \Omega$, making use of the asymptotic form $I_{0}(z) \simeq(2 \pi z)^{-1 / 2} \exp (z)$ and the condition

$$
\omega=\frac{d \chi}{d b}=\mu \beta K_{1}(\mu b) \ll \Omega
$$

yields

$$
\begin{aligned}
\langle\exp (i \chi)\rangle \simeq 2 \Omega \int \frac{s d s}{\sqrt{\pi b s}} \exp [ & \left.-\Omega^{2}(b-s)^{2}\right] \exp [i \chi(\mu s)] \\
& \simeq \exp (i \chi) \exp \left[-\omega^{2} / 4 \Omega^{2}\right]
\end{aligned}
$$

From $(11,5,1)$ it follows that the absorption is especially strong for impact parameters 


$$
b \lesssim b_{a}=\frac{1}{2 \mu} \log \frac{\pi \mu^{2} \beta^{2} N}{4 \Omega^{2}} .
$$

For $b \lesssim b_{a}$ the atomic chain acts like an opaque "black" disc. Certainly, the value of this finding differs for different observables and for different processes proceeding at different impact parameters.

Integrating once by parts reduces $F(q)$ to the form convenient for evaluation of the total cross section,

$$
F(q)=\frac{i p \mu N}{q} \int_{0}^{\infty} b d b J_{1}(q b)\left\langle i \chi^{\prime} \exp (i \chi)\right\rangle\langle\exp (i \chi)\rangle^{(N-1)}
$$

At small $q$ and large $N$ only large impact parameters, $b \gg \mu^{-1}$, may contribute to $F(q)$. This is the multiple scattering effect $[1,3]$ which gives rise to the dominance of ultra-peripheral collisions in the coherent particle-crystal interactions. Then, for $q \lesssim q_{0}=\mu / \xi$ and $\xi \gg 1$, the steepest descent from the saddle-point

$$
b_{0}=\mu^{-1}[\xi+i \pi / 2]
$$

in eq.(13) yields

$$
F(q) \simeq \frac{i p b_{0}}{q} J_{1}\left(q b_{0}\right)
$$

The effect of lattice thermal vibrations at small $q$ appears to be marginal and reduces to the factor $\exp \left(\mu^{2} / 4 \Omega^{2} N\right)$ in (15) which is irrelevant in the region of large $N$ where the amplitude $F(q)$ coincides with the elastic scattering amplitude given by the SL approximation [3].

If $q \gtrsim q_{0}$ the stationary phase approximation gives the elastic scattering amplitude of the form

$$
F(q) \simeq \frac{-i p \sqrt{\eta}}{\mu q} \exp \left(-\frac{i q \eta}{\mu}\right) \exp \left(-\frac{q^{2}}{4 \Omega^{2} N}\right)
$$

where $\eta=\log (\mu \beta N / q) \gg 1$. This is the lattice vibrations which provide a natural momentum cutoff and insure the convergence of the integral for the coherent elastic scattering cross section,

$$
\begin{aligned}
\sigma_{e l} & =\frac{\pi}{p^{2}} \int d q^{2}|F(q)|^{2} \approx \frac{\pi \xi^{2}}{\mu^{2}} \int_{0}^{q_{0}^{2}} \frac{d q^{2}}{q^{2}} J_{1}^{2}\left(\frac{q \xi}{\mu}\right) \\
& +\frac{\pi}{\mu^{2}} \int_{q_{0}^{2}}^{\infty} \frac{d q^{2}}{q^{2}} \log \left(\frac{\mu \beta N}{q}\right) \exp \left(-\frac{q^{2}}{2 \Omega^{2} N}\right)
\end{aligned}
$$

which for $\xi \gg 1$ is simply

$$
\sigma_{e l} \approx \frac{\pi}{\mu^{2}} \xi^{2}
$$

From eq.(15) by means of the optical theorem we find the total cross section 


$$
\sigma_{t o t}=\frac{4 \pi}{p} \operatorname{Im} F(0) \approx \frac{2 \pi}{\mu^{2}} \xi^{2}
$$

Consequently, at high-energy and for $\xi \gg 1, \sigma_{e l} \approx \frac{1}{2} \sigma_{t o t}$.

Now let us turn to the process of the coherent Coulomb excitation of ultra-relativistic particles and nuclei passing through the crystal. This way of the experimental study of rare processes has been proposed in $[4,5,6,7,8,9,10]$.

The ultra-relativistic projectile-nucleus (the mass number $A$, the charge $Z_{1}$ and the fourmomentum $p$ ) moving along a crystal axis undergoes a correlated series of soft collisions which give rise to diagonal $\left(A \rightarrow A, A^{*} \rightarrow A^{*}\right)$ and off-diagonal $\left(A \rightarrow A^{*}, A^{*} \rightarrow A\right)$ transitions. In $[9,4,5]$ it has been proposed to study the electric dipole transition in ${ }^{19} F$, the excitation of the state $\left|J^{\pi}=1 / 2^{-}\right\rangle$from the ground state $\left|1 / 2^{+}\right\rangle$. The phenomenological matrix element of the transition $1 / 2^{+} \rightarrow 1 / 2^{-}$is $[14]$

$$
\mathcal{M}=\frac{1}{2} d \bar{u}\left(p^{\prime}\right) \gamma_{5}(\hat{q} \hat{\varepsilon}-\hat{\varepsilon} \hat{q}) u(p),
$$

where both $u\left(p^{\prime}\right)$ and $u(p)$ are bispinors of initial and final states of the projectile, $d$ is the transition dipole moment and $\varepsilon$ is the photon polarization vector. The transverse and longitudinal components the 4 -vector $p-p^{\prime}$ are denoted by $\mathbf{q}$ and $\kappa$, respectively. In what follows $q=|\mathbf{q}|$. Because of large value of the life-time of the $110 \mathrm{KeV}$ level ${ }^{19} F\left(1 / 2^{-}\right)$ [13] the decay of excited state inside the target crystal can be safely neglected. Due to the smallness of the transition dipole moment, $d \simeq 5 \times 10^{-8} \mathrm{KeV}^{-1}$, the excitation amplitude is much smaller than the elastic Coulomb amplitude for all $q$ up to $q \sim \sqrt{4 \pi \alpha} Z_{1} / d$ and can be considered as a perturbation [14]. Thus, the multi-channel problem reduces to the one-channel one.

The high-energy helicity-flip Born amplitude of the transition $1 / 2^{+} \rightarrow 1 / 2^{-}$in collision of the projectile-nucleus with $N$ bound atoms in crystal reads

$$
F_{e x}^{B}(\mathbf{q})=S(\kappa) \frac{p}{2 \pi} \frac{g(\boldsymbol{\sigma} \mathbf{q})}{q^{2}+\lambda^{2}} \exp \left(-\frac{q^{2}}{4 \Omega^{2}}\right),
$$

where $\boldsymbol{\sigma}=\left(\sigma_{1}, \sigma_{2}, \sigma_{3}\right)$ is the Pauli spin vector, $\left\{\sigma_{i}, \sigma_{j}\right\}=2 \delta_{i j}$ and the amplitude we are constructed is to be regarded as an operator which transforms the initial helicity state of the projectile into its final state. In the denominator of eq.(21) $\lambda^{2}=\mu^{2}+\kappa^{2}$. In the Glauber approximation the longitudinal momentum transfer which determines the coherency length, $l_{c} \sim \kappa^{-1}$, reads $[11]$

$$
\kappa=\frac{M \Delta E}{p}
$$

where $M$ is the mass of projectile and $\Delta E$ is the excitation energy ${ }^{2}$.

The structure factor of crystal $S(\kappa)$ to the first order in $g$ is

$$
S(\kappa)=\exp \left[-\frac{\kappa^{2}}{4 \Omega^{2}}\right] \frac{\sin (\kappa N a / 2)}{\sin (\kappa a / 2)} .
$$

\footnotetext{
${ }^{2}$ The Fresnel corrections to geometrical optics which are neglected here become important for large $N$ or large $q$ diminishing the coherency length and bringing about an additional suppression of coherent processes [12].
} 
If the projectile momentum satisfies the resonance condition $[4,5,9,7]$

$$
\frac{M \Delta E}{p}=\frac{2 \pi n}{a}, n=0,1,2 \ldots
$$

$S(\kappa) \sim N$. Then, to the first order in $g$ (Born approximation) the cross section of the coherent excitation of the projectile in scattering on a chain of $N$ atoms in crystal is

$$
\sigma_{e x}^{B}=\frac{\pi}{p^{2}} \int d q^{2}\left|F_{e x}^{B}(\mathbf{q})\right|^{2} \propto \frac{g^{2} N^{2}}{4 \pi}
$$

where $g=\sqrt{4 \pi \alpha} d Z_{2}$. The central idea of $[4,5,6,7,9,10]$ based on the Born approximation is that the transition rate can be enhanced substantially due to coherency of interactions which is assumed to sustain over the large distance scale. The law $\sigma_{e x} \propto N^{2}$ is expected to hold true up to the crystal thicknesses $N=L / a \sim 10^{5}-10^{6}$ in tungsten target. In [10] the Born approximation for the coherent excitation of $\Sigma^{+}$in high-energy proton-crystal interactions $p \gamma \rightarrow \Sigma^{+}$has been assumed to be valid up to $N \sim 10^{8}$. However, the account of the initial and final state Coulomb interactions dramatically changes the dependence of $\sigma_{e x}$ on $N$. For example, even in the diamond crystal

$$
\sigma_{e x} \sim \sigma_{e x}^{B}\left(1-\frac{N \omega^{2}}{2 \Omega^{2}}\right)
$$

and $\omega^{2} / 2 \Omega^{2} \simeq 2 \beta^{2} \mu^{2} / \Omega^{2} \sim 1 / 20$ (see [15] for more details). Thus, the Born approximation becomes irrelevant already at $N \gtrsim 10$.

The evaluation of the transition amplitude on a chain of $N$ identical atoms including all the multi-photon t-channel exchanges reads

$$
F_{e x}(\mathbf{q})=\frac{p}{\pi} \int d^{2} \mathbf{b} \exp (i \mathbf{q} \mathbf{b})\left\langle f_{e x}^{B} \exp (i \chi)\right\rangle\langle\exp (i \chi)\rangle^{N-1}
$$

The eq.(27) contains two bracketed factors. The first one corresponds to the nuclear excitation amplitude in scattering on the atom bound in crystal. At small impact parameters, $b \lesssim 1 / 2 \Omega$,

$$
\left\langle f_{e x}^{B} \exp (i \chi)\right\rangle \simeq S(\kappa) \frac{g}{2 \pi b}\left(\boldsymbol{\sigma} \mathbf{n}_{\mathbf{b}}\right) \sinh \left(\frac{1}{2} \Omega^{2} b^{2}\right) \exp \left(-\frac{1}{2} \Omega^{2} b^{2}\right) .
$$

Because of both the multiple scattering effect and absorption only $b \gg \mu^{-1}$ may contribute to $F_{e x}(\mathbf{q})$. In this region of impact parameters

$$
\left\langle f_{e x}^{B} \exp (i \chi)\right\rangle \simeq S(\kappa) \frac{g}{4 \pi}\left(\boldsymbol{\sigma} \mathbf{n}_{\mathbf{b}}\right) \lambda K_{1}(\lambda b) \exp (i \chi) \exp \left(-\frac{\omega^{2}}{4 \Omega^{2}}\right) .
$$

The second factor in (27) describes the initial and final state interactions of the projectile and has been calculated above. Then,

$$
\begin{aligned}
& F_{e x}(\mathbf{q}) \approx \frac{g p}{2 \pi} S(\kappa)\left(\boldsymbol{\sigma} \mathbf{n}_{\mathbf{q}}\right) \int_{1 / \mu}^{\infty} b d b J_{1}(q b) \\
& \times \lambda K_{1}(\lambda b) \exp (i N \chi) \exp \left(-N \omega^{2} / 4 \Omega^{2}\right),
\end{aligned}
$$


where $\mathbf{n}_{\mathbf{q}}=\mathbf{q} /|\mathbf{q}|$. The contribution of the domain $q \lesssim q_{0}=\mu / \xi$ to the excitation cross section can be neglected as far as $F_{e x} \propto q$ for $q \lesssim q_{0}$. If $q \gg q_{0}$ and $\xi \gg 1$, the stationary phase approximation gives the coherent excitation amplitude of the form

$$
\begin{aligned}
F_{e x}(\mathbf{q}) \approx & \frac{i p g\left(\boldsymbol{\sigma} \mathbf{n}_{\mathbf{q}}\right)}{2 \pi \beta} \frac{S(\kappa)}{N} \frac{\lambda}{\mu} \sqrt{\eta} \exp (-\delta \eta) \\
& \times \exp \left(-\frac{i q \eta}{\mu}\right) \exp \left(-\frac{q^{2}}{4 \Omega^{2} N}\right)
\end{aligned}
$$

We see that the helicity-flip dynamics removes the factor $1 / q$ from the elastic amplitude (16) thus making the UV-regularization of the excitation cross section indispensable. The latter is evaluated as,

$$
\begin{gathered}
\sigma_{e x}=\frac{\pi}{p^{2}} \int d q^{2}\left|F_{e x}(\mathbf{q})\right|^{2} \\
\sim \frac{g^{2} N^{1-\delta}}{8 \pi} C \log \left(\frac{N}{\delta \gamma}\right),
\end{gathered}
$$

where $C=\gamma^{\Delta} \Delta^{2} \Gamma(\Delta), \gamma=2 \Omega^{2} / \beta^{2} \mu^{2}, \Delta=\lambda / \mu$ and $\delta=\Delta-1 \sim \kappa^{2} / 2 \mu^{2} \ll 1$. In (32) we put simply $S(\kappa)=N$. Thus, the account of multiple scatterings and absorption turns the Born approximation cross section $\sigma_{e x} \propto N^{2}$ into $\sigma_{e x} \propto N^{1-\delta} \log N$. In the limit of $p \rightarrow \infty$ and $\delta \rightarrow 0$

$$
\sigma_{e x} \sim \frac{g^{2} N}{8 \pi} \gamma \log \left(\frac{N}{\gamma}\right)
$$

The dependence of $\sigma_{e x}$ on $N$ differs from that of fully unitarized $\sigma_{e l} \propto \log ^{2} N$. The reason is that in $\sigma_{e x}$ we sum the eikonal diagrams to all orders in $\beta$ but only to the first order in $g$. Such a unitarization procedure is, of course, incomplete, but this is of no importance for practical purposes since the smallness of $d^{2} \Omega^{2}$ makes the next to leading order corrections negligibly small up to $N \sim \alpha Z_{1}^{2} / \delta \Omega^{2} d^{2} \sim 10^{12}$.

Acknowledgments: Thanks are due to N.N. Nikolaev for useful comments.

\section{References}

[1] R.J. Glauber, in Lectures in Theoretical Physics, edited by W.E. Brittin et al., Interscience Publishers, Inc., New York, vol.1, p. 315, 1959.

[2] D.S. Gemmel, Rev. Mod. Phys. 46 (1974) 1.

[3] N.P.Kalashnikov, E.A.Koptelov and M.I.Ryazanov, Sov. Phys. JETP 63 (1972) 1107; N.P.Kalashnikov and V.D.Mur, Sov. J. Nucl. Phys. 16 (1973) 613.

[4] V.V. Okorokov, Sov. J. Nucl. Phys. 2 (1966) 719; V.V. Okorokov, Yu.L. Pivovarov, A.A. Shirokov, S.A. Vorobev, Proposal of experiment on coherent excitation of relativistic nuclei in crystals, Moscow, ITEP-90-49, Fermilab Library Only. 
[5] V.V. Okorokov, S.V. Proshin, Investigation of the coherent excitation of the relativistic nuclei in a crystal, Moscow, ITEP-13-1980

[6] Yu.L. Pivovarov, H. Geissel, Yu.M. Filimonov, O.E. Krivosheev, C.Scheidenberger, On the resonant coherent excitation of relativistic heavy ions GSI-95-38, Darmstadt, Jul 1995

[7] Yu.L. Pivovarov and A.A. Shirokov, Sov. J. Nucl. Phys. 37 (1983) 653

[8] Yu.L. Pivovarov, A.A. Shirokov, S.A. Vorobev, Nucl.Phys. A509 (1990) 800

[9] R. Fusina, J.C. Kimball, Nucl.Instrum.Meth. B33 (1988) 77

[10] A. Dubin, Sov.J.Nucl.Phys.52:790-793,1990, Yad.Fiz.52:1243-1249,1990

[11] V.N. Gribov, Sov. Phys. JETP 29 (1969) 483; 30 (1970) 709.

[12] V.R. Zoller, Phys.Lett. B416 (1998) 447

[13] F. Ajzenberg-Selove, Nucl. Phys A190 (1972) 1.

[14] V.R. Zoller, JETP Lett. 75 (2002) 119 ; Pis'ma v ZhETP 75 (2002) 147.

[15] V.R. Zoller, Ultra-Relativistic Nuclei in Crystal Channel: Coulomb Scattering, Coherence and Absorption, ITEP-PH-2/2004 